\title{
ANALYSIS OF LASER ULTRASONIC MEASUREMENTS OF SURFACE WAVES ON
}

\section{ELASTIC SPHERES}

\author{
I.. S. Koo and K. L. Telschow \\ Idaho National Engineering Laboratory \\ EG\&G Idaho, Inc. \\ P.O. Box 1625 \\ Idaho Falls, ID 83415-2209
}

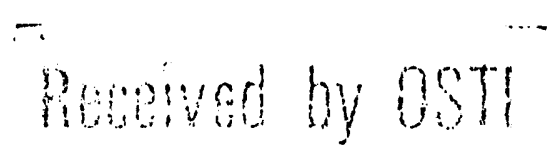

NOV 221991

\section{INTRODUCTION}

In conventional ultrasonic nondestructive evaluation studies, piezoelectric trainsducers are used to generate sound waves in solids via a couplant that transmits the mechanical motions. In recent years, a different method of generating sound in solids, pulsed laser heating, was introduced by White $[1,2]$. This method is noncontacting, requires no coupling medium, and operates directly on the surface of the specimen. Noncontacting ultrasonic detection using laser interferometers of several types has also been developed [3]. Laser techniques can achieve essentially point source and point detection of ultrasonic motion tirrough focusing. Laser ultrasonics can, therefore, be used on objects with complex shapes, e.g. curved surfaces, and are applicable to material shapes more commonly found in industry. Often the goal of ultrasonic measurements is to determine material properties such as Lame's elastic constants. The conventional approach measures longitudinal and shear wave speeds between two parallel flat surfaces. The work reported here demonstrates the versatility of laser ultrasonics by directly measuring the surface motion of a solid sphere generated by ablation from a pulsed laser beam in the ablation regime. The results compare well with elastodynamic theoretical calculations, where the ablation source is approximated as a normal impulse on the surface. This work suggests that an algorithm could be formulated to measure elastic properties of targets with curved surfaces.

\section{EXPERIMENTAL MEASUREMENTS}

A pulsed laser can generate ultrasonic waves in a target by two methods: the creation of a transient subsurface thermal expansion (thermoelastic) and surface evaporation (ablation) [4-7]. In this study, a volatile liquid coating was applied to the surface of a sphere and ablated by the laser pulse. This method produces a strong ablation source without causing surface damage. The experimental measurements of surface waves on a methanol-coated type 304 stainless steel sphere are shown in Fig. 1. The out-of-plane motion (radial velocity component at the surface) was measured with a confocal Fabry-Perot interferometer $[3,8]$. Detailed discussions of the experimental methodology can be found in reference [8].

A similar study [9] reported measurements of Rayleigh waves generated on a sphere by a thermoelastic laser source and illustrated the general properties of this wave (e.g. the focusing at the poles and the wave dispersion). In the present work, the complete wave train of the forward-focusing group is measured (Fig. 1) and analyzed theoretically. This group of waves is bounded by the earliest arriving wave $L_{1}$ and the first Rayleigh wave $R_{1}$ (the 


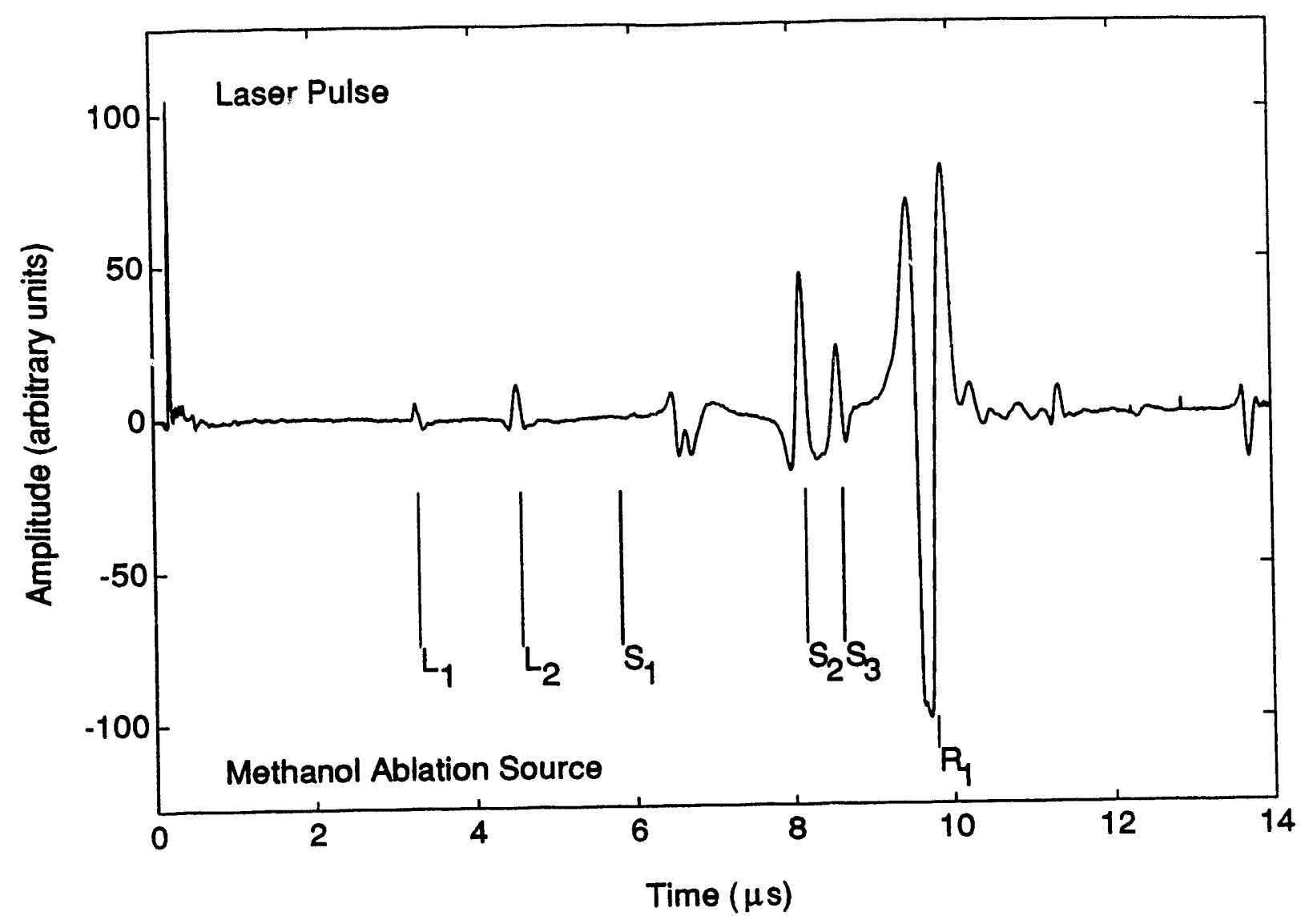

Fig. 1. Measurements of ultrasonic waves at the detecting pole of the target sphere.

strongest resionse in Fig. 1). All the other waves after $R_{1}$ are not part of the forwardfocusing group. The radius of the solid sphere was $8.73 \mathrm{~mm}$. The laser source and the detecting laser interferometer were placed at opposite poles as is illustrated in Fig. 2. As can be seen from the geometry, and as is shown in Fig. 1, the earliest arrival at the detecting pole is the longitudinal wave directly traversing the sphere $\left(\mathrm{L}_{1}\right)$. Based on the theoretical calculations of angular directivity given in [4], there is no shear wave expected following the path of $L_{1}$, as confirmed in Fig. 1 . All the other rays, bounded by $L_{1}$ and $R_{1}$, are due to multiple internal reflections with or without wave mode conversions at the surface. The waves due to internal multiple boundary reflection have been historically called "whispering gallery" waves [10]. For rays of the whispering gallery group that do not have wave mode conversion, that are either pure longitudinal $\left(\mathrm{L}_{1}\right)$ or pure shear $\left(\mathrm{S}_{1}\right)$ from the source pole to the detecting pole, the ray paths $p_{1}(1=1,2,3, \ldots)$ can be calculated from the geometry by

$p_{l}=l a \sqrt{2\left[1-\cos \left(\frac{\pi}{l}\right)\right]}, \quad$ where (a) is the sphere radius and $l=1,2,3, \ldots$.

Therefore the time-of-flight $t_{1}$ of $L_{1}\left(\right.$ or $\left.S_{1}\right)$ is

$t_{l}=\frac{p_{l}}{C}$

where the wave speed $C$ is either the longitudinal wave speed, $C_{L}$, or the shear wave speed, $C_{S}$. Some identified waves $\left(\mathrm{L}_{2}, \mathrm{~S}_{2}, \mathrm{~S}_{3}\right)$ are indicated in Fig. 1 and Fig. 2 . The other waves are probably due to wave mode conversions and critical angle surface reflections. The arrival time of the Rayleigh wave at any surface point can be approximately computed by using the half-space Rayleigh wave speed, $C_{R}$, 


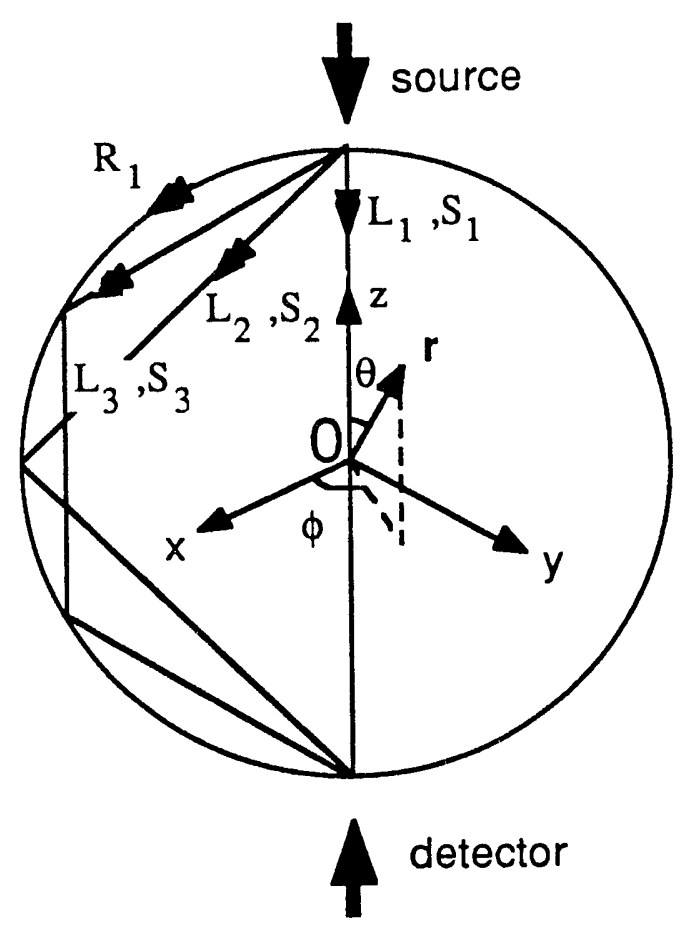

Fig. 2. Schematic experimental configuration and some geometrical ray paths.

$t_{R}=\frac{a \theta}{C_{R}}$

Similarly to the thick plate case considered theoretically in [11], the Rayleigh wave on a spherical surface is strongest while the longitudinal wave is weakest. The ratio of any two strengths among the longitudinal, shear, and Rayleigh waves depends on Poisson's ratio. It is known that the spherical Rayleigh wave is dispersive due to the surface curvature [9]. As seen in Fig. 1, after having traveled half of the spherical surface the Rayleigh wave shows a strong resemblance to the analogous flat surface half-space waveform [4] and exhibits little change in waveform due to dispersion; however, after several passes around the sphere significant dispersive effects were observed.

\section{THEORETICAL ANALYSIS}

In this section, the development follows closely the work of Satô and Usami $[12,13]$. The technique is based on Fourier synthesis and summation of normal modes that are specified by the combination of indices $\mathrm{n}$ and $\mathrm{i}$ as discussed in the following. To model the ablation effect, a normal stress depending on both the polar angle, $\theta$, (Fig. 2) and time, $t$, is assumed at the source pole on the surface together with zero surface shear stresses

$$
\begin{aligned}
& \left.\sigma_{r r}\right|_{r=a}=\Phi(\theta) F(t) \\
& \sigma_{r} d_{r=a}=\left.\sigma_{r}\right|_{r=a}=0 .
\end{aligned}
$$


Due to the axial symmetry, the responding motions can be spheroidal only and hence do not depend on the azimuthal angle, $\phi$. Harmonic fields are assumed to calculate the displacement components and then the Helmoltz equations are solved using the standard technique of separation of variables [14]. With Fourier synthesis, the harmonic fields were summed to obtain the time domain functions. Following this outlined method, the surface radial displacement can be written as

$u_{r}(r, t)=\frac{1}{2 \pi} \sum_{n} P_{n}(\cos \theta) D_{n} \int_{-\infty}^{\infty} \frac{U_{n}(r, \omega)}{E(\omega)} f(\omega) e^{j \omega t} d \omega \quad$ evaluated at $\mathrm{r}=\mathrm{a}$

where $f(\omega)$ is the Fourier transform of $F(t), P_{n}(\cos \theta)$ is the Legendre polynomial with $\mathrm{n}=0,1,2, \ldots$ and

$U_{n}(r)=\mu\left[\sqrt{\frac{k}{h}}\left(j_{n}^{\prime \prime}(k r)+(n(n+1)-2) \frac{j_{n}(k r)}{(k r)^{2}}\right) j_{n}^{\prime}(h r)-\sqrt{\frac{h}{k}} 2 n(n+1) \frac{d\left(j_{n}(h r) /(h r)\right)}{d(h r)} \cdot \frac{j_{n}(k r)}{k r}\right]$

$D_{n}=\frac{2 n+1}{4 \pi} \int_{-1}^{1} \Phi(\theta) P_{n}(\cos \theta) d(\cos \theta)$

with $j_{n}$ denoting the nth order spherical Bessel function of the first kind and $h, k$ being the longitudinal and shear wave numbers respectively. The primes in the above equations denote differentiations with respect to the argument. The denominator $E$ is

$$
\begin{gathered}
E=\mu j_{n}(\xi) j_{n}(\eta)\left[\frac{2 n(n-1)-\eta^{2}}{\xi^{2}}+\frac{4}{\xi} \cdot \frac{j_{n+1}(\xi)}{j_{n}(\xi)}\right] \cdot\left[\frac{2\left(n^{2}-1\right)-\eta^{2}}{\eta^{2}}+\frac{2}{\eta} \cdot \frac{j_{n+1}(\eta)}{j_{n}(\eta)}\right]- \\
4 n(n+1) \mu j_{n}(\xi) j_{n}(\eta)\left[\frac{n-1}{\xi^{2}}+\frac{1}{\xi} \cdot \frac{j_{n+1}(\xi)}{j_{n}(\xi)}\right] \cdot\left[\frac{n-1}{\eta^{2}}+\frac{1}{\eta} \cdot \frac{j_{n+1}(\eta)}{j_{n}(\eta)}\right]
\end{gathered}
$$

which has only simple poles. At these poles, $E=0$, which gives the normal mode frequencies of a traction-free sphere.

In the above equations, the longitudinal or shear dimensionless frequencies on the surface are denoted as $\xi$ or $\eta$ respectively where $\xi=$ ha and $\eta=k a$. Instead of using direct integration to evaluate (5), contour integration is more desirable and yields

$u_{r}(a, t)=j \sum_{n, i} P_{n}(\cos \theta) D_{n}\left[\frac{U_{n}\left(a, \omega_{n, i}\right)}{\frac{d E}{d \omega}\left(\omega_{n, i}\right)} f\left(\omega_{n, i}\right) e^{\left.j \omega_{n, i}\right]}\right]$

where $\omega_{n, i}$ are the simple poles of $E$. As was shown in [13], the fundamental mode $i=1$ and $\mathrm{n}>0$ forms the Rayleigh wave. For $\mathrm{n}=0$, the sphere vibrates symmetrically with respect to its center and hence these motions are denoted as the compressional modes. The modes with $i>1$ and $n>0$ give rise to all the other internal reflections including the whispering gallery phenomenon. Fig. 3 shows the dispersion relation for the spheroidal modes in terms of the normalized shear frequency $(\eta)$. The compressional modes $(n=0)$ are plotted as $x$ 's along the vertical axis. The Rayleigh modes $(i=1$ and $n>0)$ are plotted as "plus" signs and the whispering gallery modes ( $i>1$ and $n>0$ ) are depicted as circles. These normal mode curves, except when $n=0$, show undulation for higher modes at small values of $n$. This irregularity is directly related to the corresponding group velocity [13]. The summations of these three groups of modes, first individually and then together, are demonstrated in Figs. 4 and 5. 


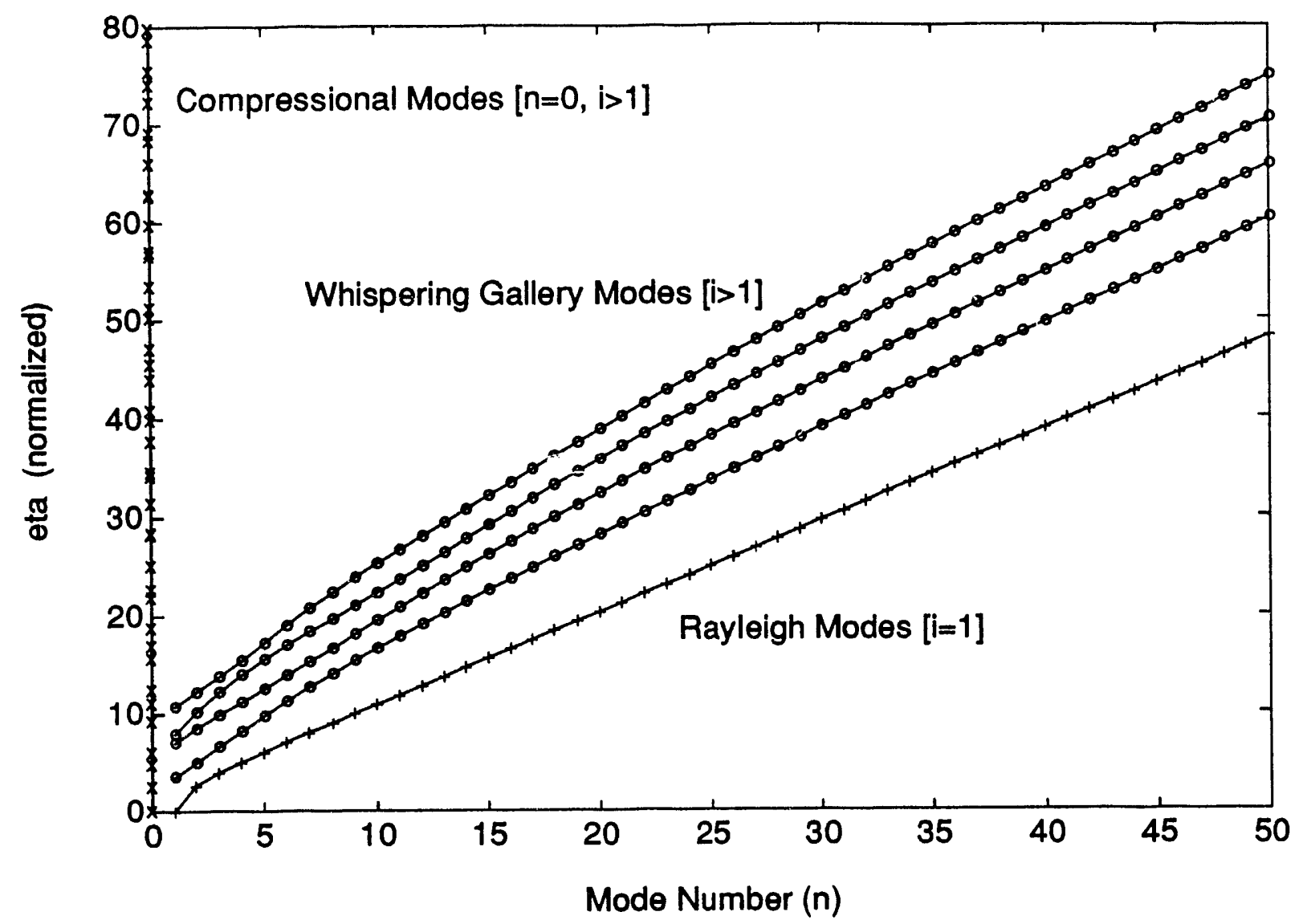

Fig. 3. Dimensionless frequency $\eta$, of the major groups of normal modes: Compressional, Whispering Gallery and Rayleigh.

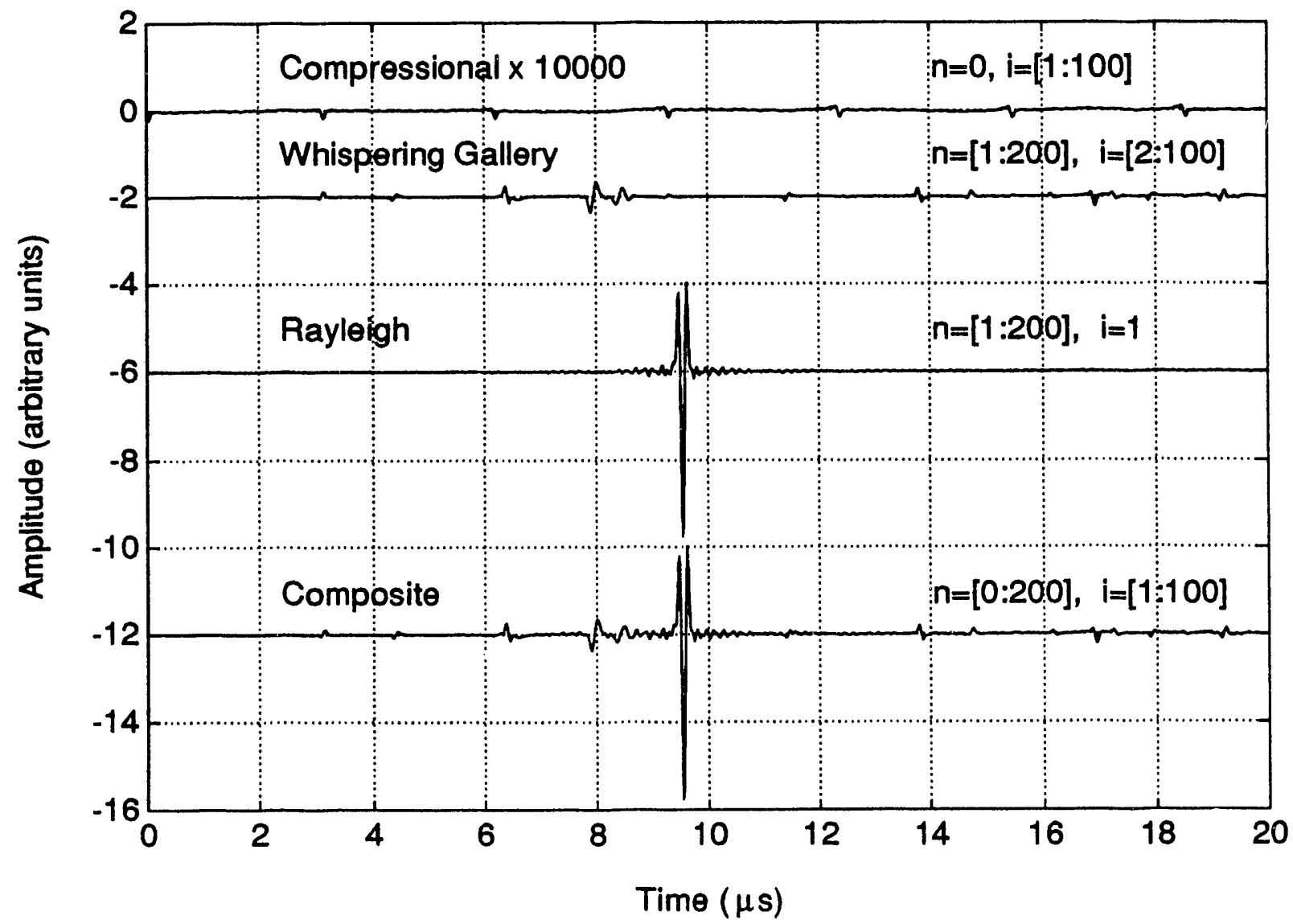

Fig. 4. Theoretical synthesis of the normal modes where the range of values for $n$ and $i$ are shown in brackets. 


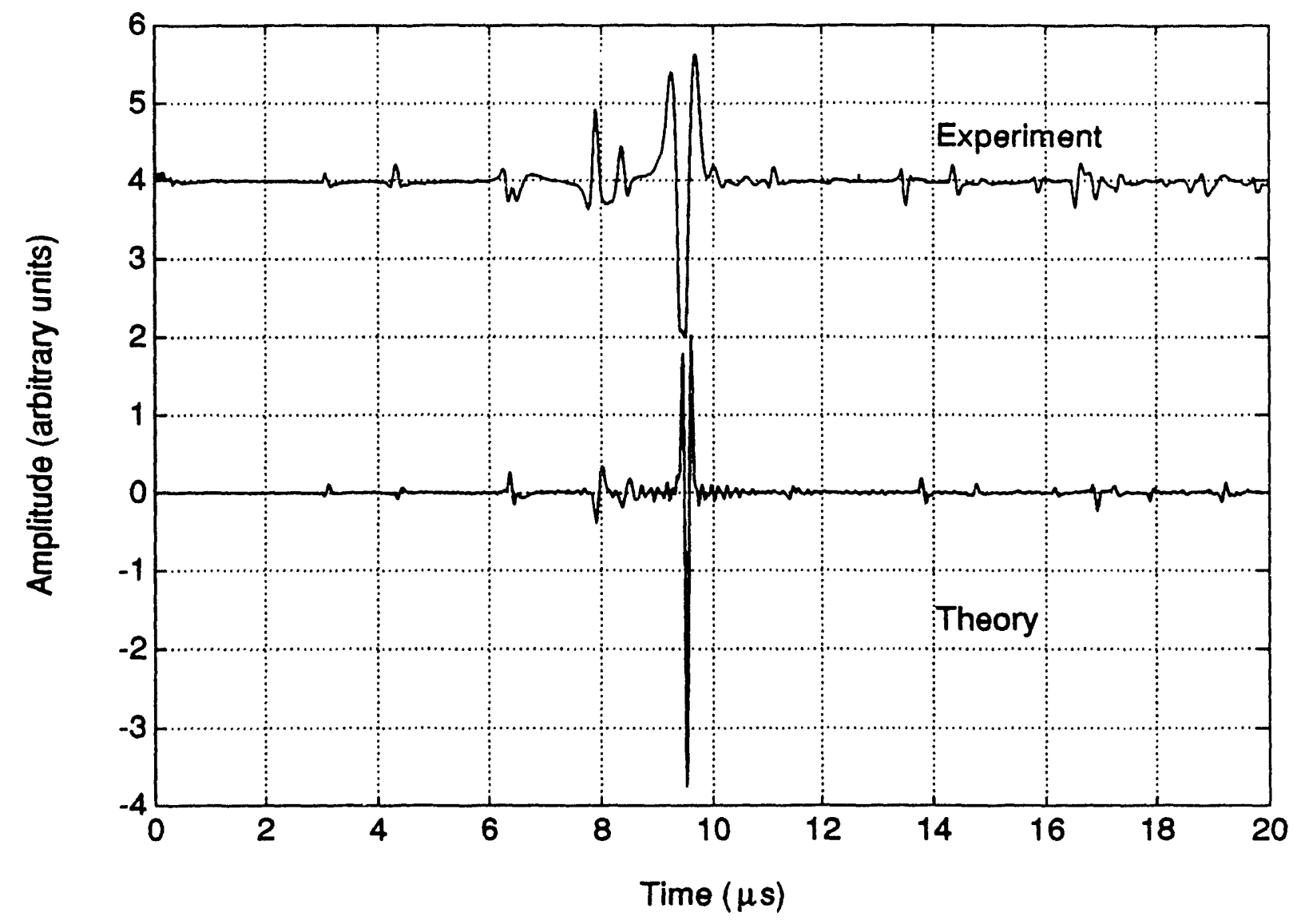

Fig. 5. Comparison of experimental and theoretical results.

In these figures, all modes with $\eta \leq 200$ were used ( $\mathrm{n} \leq 200, \mathrm{i} \leq 100$ ) and only the radial surface velocity motion is plotted as this approximates the experimental measurements [8]. To compare the experimental and the theoretical results, both the calculated composite wave train and the experimental measurements are repeated in Fig. 5. Other than the mismatch of some wave forms (profiles) and a slight time delay in the theoretical predictions, the computational result agrees well with the measured result. The slight discrepancy in arrival times is due to the phase velocities $C_{L}(=5.66 \mathrm{~mm} / \mu \mathrm{s})$ and $C_{S}(=3.12 \mathrm{~mm} / \mu \mathrm{s})$ used in the calculation, which were taken from standard elastic values rather than direct measurements of the target. Since the source laser pulse width was only $10 \mathrm{~ns}$, a Dirac delta function in time dependence was used in Eq. 4a. The spatial extent of the boundary loading determines the coefficients $D_{n}$ as given in Eq. 7. These coefficients are proportional to a mode number for small $n$, reach a maximum, and eventually decrease for large $n$. This pattern produces a spatial loading function that decreases rapidly with polar angle $(\theta)$. Such a function, with an angular width of about 5 degrees, was used to produce the calculated results shown in Figs. 4 and 5. Also, the response of the Fabry-Perot interferometer was approximated according to reference [15]. Both the interferometer response and the source angular distribution are not well known; this uncertainty may be the cause of the difference in bandwidth between the experimental and theoretical results displayed in Fig. 5.

\section{CONCLUSION AND FUTURE RESEARCH}

The good agreement between experimental measurement and theoretical calculation demonstrates the utility of laser ultrasonics for materials with curved surface geometries, such as a sphere. Even though there are no simple nondispersive waveforms present with 
which to determine material elastic constants for the sphere, close comparison between analytic calculations and experimental measurements could lead to an accurate scheme for elastic constant determination. This scheme would involve comparison of many waveforms (such as the whispering gallery waves) simultaneously.

\section{ACKNOWLEDGMENTS}

This work was supported by the Interior Department's Bureau of Mines under Contract No. J0134035 through Department of Energy Contract No. DE-AC07-76ID01570.

\section{REFERENCES}

1. R. M. White, J. of Appl. Phys. 34 2123 (1963).

2. R. M. White, J. of Appl. Phys. 34,3559 (1963).

3. J. P. Monchalin, IEEE Trans. UFFC 33, 485 (1986).

4. C. B. Scruby, R. J. Dewhurst, D. A. Hutchins and S. B. Palmer, in Research Techniques in Nondestructive Testing, vol. 5, edited by R. S. Sharpe (Academic Press, New York, 1982), p. 281.

5. U. Schleichert, K. J. Langenberg, W. Amold and S. Fabbender, in Review of Progress in QNDE, vol. 8A, edited by D. O. Thompson and D. E. Chimenti (Plenum Press, New York, 1989), p. 489.

6. J. D. Aussel, A. Le Brun and J. C. Baboux, Ultrason. 26, 245 (1988).

7. K. L. Telschow and R. J. Conant, J. Acoust. Soc. Am. 88,1494 (1990).

8. K. L. Telschow, J. B. Walter and G. V. Garcia, J. of Appl. Phys. 68 , 6077 (1990).

9. D. Royer, E. Dieulesaint, X. Jia and Y. Shui, Appl. Phys. Lett. 52, 706 (1988).

10. J. W. S. Rayleigh, The Theory of Sound, vol. 2, 2 ed. (Dover, New York, 1945).

11. L. R. F. Rose, J. Acoust. Soc. Am. 75, 723 (1984).

12. Y. Satô and T. Usami, Geophy. Mag. 31, 25 (1962).

13. T. Usami and Y. Satô, Bull. Earthquake Res. Inst. (Tokyo) 42, 273 (1964).

14. P. M. Morse and H. Feshbach, Methods of Theoretical Physics, part 2 (McGrawHill, New York, 1953).

15. J. P. Monchalin and R. Heon, Mat. Eval. 44, 1231 (1986).

\section{DISCEAIMER}

This report was prepared as an account of work sponsored by an agency of the United States Government. Neither the United States Government nor any agency thereof, nor any of their (iability or responsiemployees, makes any warranty, express or implied, or assumes any legal lion, apparatus, product, or bility for the accuracy, completeness, or usefulness of any information, appatalus, product, or process disclosed, or represents that its use would not inf re price by trade name, trademark, ence herein to any specific commercial product, process, or service by trade name, trademark, manufacturer, or otherwise does not necessarily constitute or imply its endorsement, recommendation, or favoring by the United States Government or any agency thereor. The views and opinions of authors expressed herein do not necessarily state or reflect those of the United States Government or any agency ihereof. 

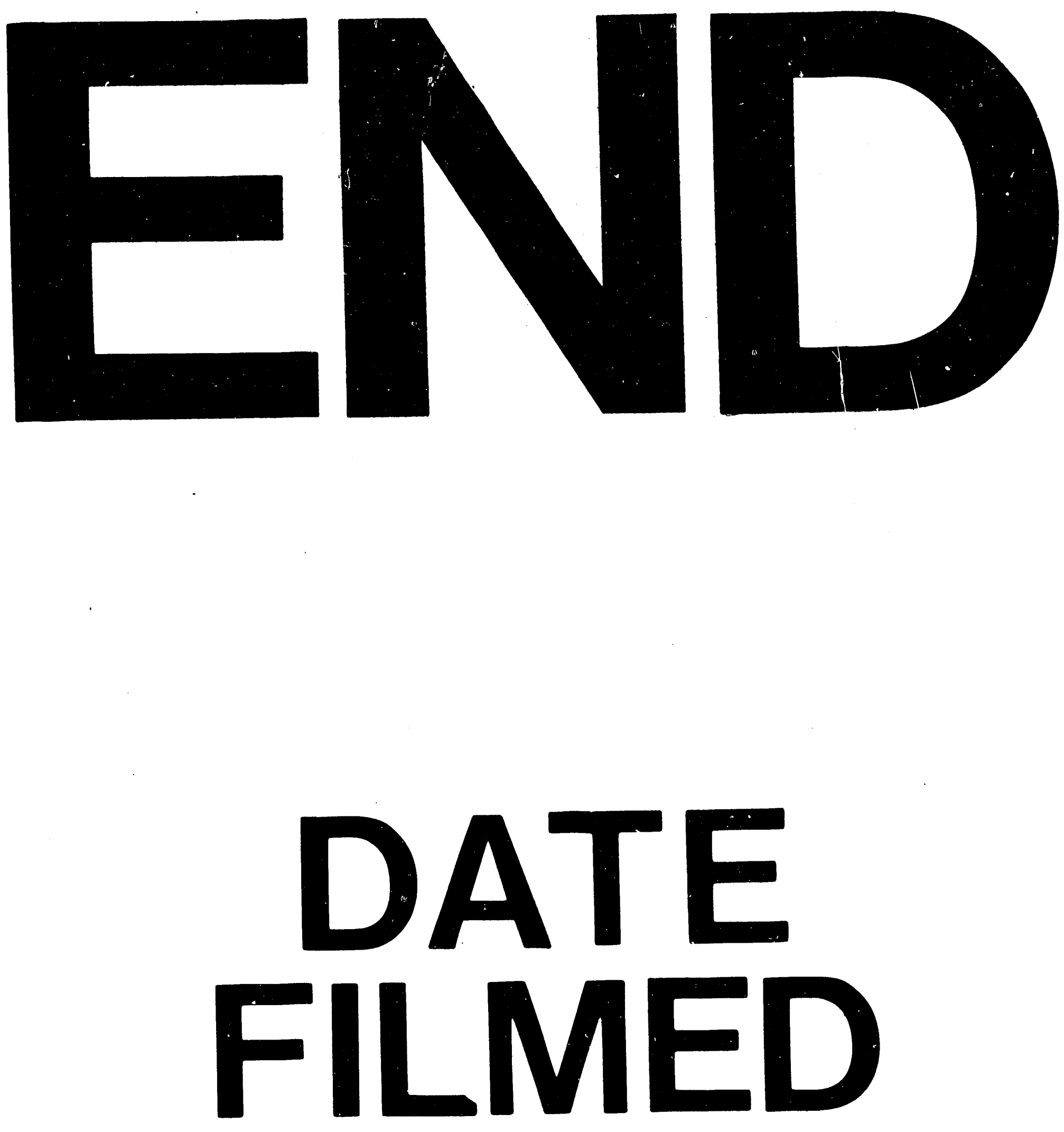

I

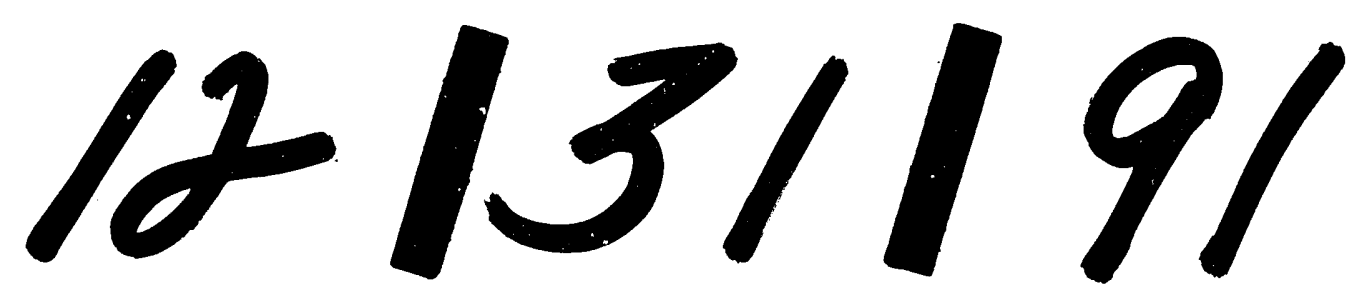


